\title{
Distributed fiber sensing using Brillouin echoes
}

\author{
Luc Thévenaz ${ }^{*}$, Stella Foaleng Mafang \\ Laboratory of Metrology and Photonics, EPFL Swiss Federal Institute of Technology, \\ STI NAM Station 11, CH-1015 Lausanne, Switzerland.
}

\begin{abstract}
A simple physical description of the nonlinear optical interaction based on Brillouin echoes is presented. This technique makes potentially possible distributed Brillouin sensing down to centimeter spatial resolution while preserving the narrowband feature of the natural Brillouin gain spectrum. Experimental conditions for the generation of Brillouin echoes are described and demonstrations of distributed measurements using a $1 \mathrm{~ns}(10 \mathrm{~cm})$ pulse are presented.
\end{abstract}

Keywords: Brillouin fiber sensing, distributed fiber sensor, stimulated Brillouin scattering.

\section{INTRODUCTION}

Distributed Brillouin fiber sensing was proposed in the late 1980 's ${ }^{1}$ for distributed temperature and strain measurements and has since evolved towards a high performance instrumentation that can achieve 1 meter spatial resolution over more than $30 \mathrm{~km}$ together with a $1 \mathrm{MHz}$ accuracy on the determination of the local Brillouin shift ${ }^{2,3}$. It was commonly accepted at that moment that the spatial resolution was ultimately limited to $\sim 1$ meter, corresponding to a pump pulse width of $10 \mathrm{~ns}$. This figure actually corresponds to the acoustic lifetime in silica that determines the Brillouin resonance linewidth. For pulses shorter than this acoustic lifetime the optical spectrum of the pump spans over a frequency range broader than the Brillouin resonance. The effective Brillouin gain spectrum then corresponds to the convolution between the pump pulse spectrum and the natural Brillouin gain spectrum ${ }^{4}$.

This commonly accepted opinion was seriously questioned when Bao et $a l^{5}$ observed an unexpected narrowing of the Brillouin gain spectrum down to its natural linewidth when pulses turned shorter than the acoustic lifetime (10 ns). This breakthrough opened the incredible perspective of performing high spatial resolution measurement while maintaining a sharp Brillouin resonance. This was actually reported with pulse width down to $1.5 \mathrm{~ns}(15 \mathrm{~cm} \text { spatial resolution })^{6}$.

Despite the unquestionable experimental demonstration brought by Bao et al these results have so far suffered from the problem that they could not be decisively reproduced with a setup different to the original (same sources, same electrooptic modulator) and by an independent team. In particular nobody could observe this spectral narrowing at $1550 \mathrm{~nm}$ using semiconductor lasers. Moreover no clear physical description of the experimental situation has been reported and the authors have so far justified their observations through a numerical integration of the coupled wave equations ${ }^{7,8}$.

It is now unanimously accepted by all specialists that this special behavior results from a pre-excitation of the acoustic wave through the presence of a continuous background co-propagating with the pump at the same optical frequency. This was originally accidentally observed by using a modulator with a poor off-state extinction ratio to form the pump pulses. A good physical insight would definitely help to understand the mechanisms behind the experiment, to determine for what reasons the experiment could not be easily replicated and, mostly important, to follow a convergent procedure toward the optimization of the configuration. Recently a very enlightening experimental approach was proposed by Horigushi et $a l^{9}$. They could achieve a very similar behavior using 2 successive pump pulses, a long weak pulse to preexcite the acoustic wave followed rapidly by a short high power pulse that experienced a $\pi$ optical phase shift.

We here present a physical description on the behavior of the Brillouin interaction using short pulses and the preexcitation of the acoustic wave. This description allowed us to rapidly conceive an experimental configuration optimizing this type of interaction, so that we could immediately obtain distributed measurement with a record high spatial resolution of $5 \mathrm{~cm}$ and a narrow Brillouin spectral resonance. This could be achieved at $1550 \mathrm{~nm}$ using semiconductor lasers. Since this type of interaction is formally and physically very similar to experiments of nuclear magnetic resonance with flipping spin fields induced by a resonant magnetic field - the so-called "spin echoes" - we find appropriate to highlight the similarity and to call this kind of interaction Brillouin Echo Distributed Sensing (BEDS).

"Email: Luc.Thevenaz@EPFL.CH; phone +41 21693 4774; fax +41 216932614 


\section{PHYSICAL DESCRIPTION}

The observation of Brillouin echoes relies on the pre-existence of an important acoustic wave in the fiber medium vibrating at the exact Brillouin frequency. This acoustic wave is conveniently generated through stimulated Brillouin scattering using a $\mathrm{CW}$ wave at the probe frequency and another $\mathrm{CW}$ wave (or a long pulse) at the pump frequency. A Brillouin echo is then observed by inducing a rapid change of the amplitude of the acoustic wave that affects the instantaneous amount of coupling between pump and probe. The most efficient way to induce this rapid change on the acoustic wave is to quench it using an out-of-phase excitation, just like resonantly knocking a pendulum during its back swing. Optically it is realized by briefly generating a $\pi$ phase shift on either the pump or the probe wave, so that the electrostriction turns antiresonant with the acoustic wave, resulting in an efficient quenching of the acoustic wave. When the phase shift returns to zero, the acoustic wave grows again and gradually restores to its former amplitude.

This is the qualitative basic description of the conditions to observe Brillouin echoes. To give a more quantitative description, let first express the 3 coupled equations governing the stimulated Brillouin interaction:

$$
\frac{\partial A_{p}}{\partial z}-\frac{1}{V_{g}} \frac{\partial A_{p}}{\partial t}=+\frac{1}{2} g_{B} A_{s} Q \quad \text { (1) } \quad \frac{\partial A_{s}}{\partial z}+\frac{1}{V_{g}} \frac{\partial A_{s}}{\partial t}=-\frac{1}{2} g_{B} A_{p} Q^{*} \quad \text { (2) } \quad \frac{\partial Q}{\partial t}+\Gamma Q=A_{p} A_{s}^{*}
$$

where $A_{p}$ and $A_{s}$ are the envelopes of the pump and the contrapropagating Stokes waves, respectively, $V_{g}$ the light group velocity, $g_{B}$ the Brillouin gain coefficient, $\Gamma$ the acoustic loss coefficient related to the acoustic lifetime by $\tau_{A}=1 / \Gamma$. In this set of equations, the optical loss and the propagation velocity of the acoustic wave are neglected and the amplitude $Q$ of the acoustic wave is normalized to electromagnetic units to simplify the expressions.

In the so-called "steady state" situation, when the optical signals vary on a longer time scale than the acoustic lifetime, the acoustic wave is supposed to vary instantaneously with a good approximation to any change of the optical amplitudes and the time derivative in (3) can be neglected. The amplitude $Q$ can be then calculated from (3) and substituted into (1) and (2) to end up with the usual set of 2 coupled equations describing the Brillouin amplification in standard conditions.

For rapidly changing optical signals the instantaneous change of the acoustic wave can no longer be assumed and the 3 equations must be solved together numerically. This makes the intuitive evaluation of the way the waves are interacting nearly impossible. To make this intuitive approach possible in order to determine the right experimental conditions, we made the following assumptions:

1) The amplitudes $A_{p}$ and $A_{s}$ are not changed significantly by the interaction and can be kept constant in the right terms of Eq. (1)-(3).

2) The changes in the amplitudes and phases of $A_{p}$ and $A_{s}$ are instantaneous when produced by an external modulator.

3) The changes in the acoustic amplitude $Q$ can be considered in Eq. (1)-(2) like a perturbation.

With the assumption that the amplitudes $A_{p}$ and $A_{s}$ are constant Eq. 3 can be readily integrated to give the following general solution:

$$
Q(t)=Q_{o}+\left(\frac{A_{p} A_{s}^{*}}{\Gamma}-Q_{o}\right)\left[1-\mathrm{e}^{-\Gamma t}\right]
$$

where $Q_{o}$ is the amplitude of the acoustic wave at $t=0$. This expression simply means that the acoustic wave amplitude will change from the initial value $Q_{o}$ asymptotically to the final amplitude $A_{p} A_{s}^{*} / \Gamma$ following an exponential law with a time constant given by the acoustic lifetime $\tau_{A}$.

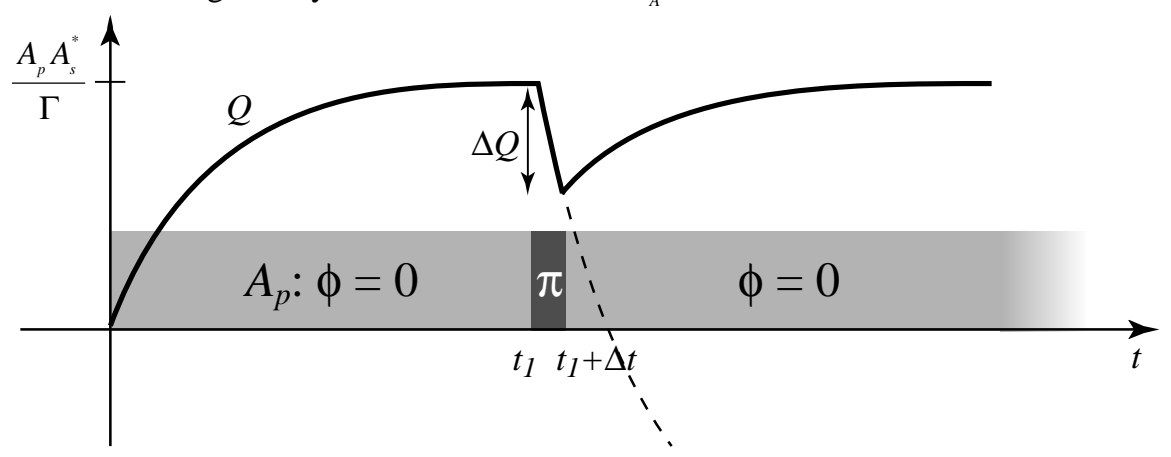

Fig. 1. Time evolution of the acoustic wave amplitude $Q$ when the optical pump wave $A_{p}$ is turned on at $t=0$, then experiences a $\pi$ phase shift at $t=t_{l}$ for a duration $\Delta t$, then returns to the initial phase. The amplitudes of pump and signal are kept constant. 
We can now sketch the situation when the pump wave experiences a sudden $\pi$ phase shift during a short time, while keep its amplitude $A_{p}$ constant. The sequence and the evolution of the acoustic wave are depicted in Fig. 1 . In the sequence the acoustic wave first grows slowly and exponentially to reach the asymptotic value $A_{p} A_{s}^{*} / \Gamma$. When the pump abruptly experiences a $\pi$ phase shift, the amplitude of the acoustic wave is rapidly quenched in a first stage since its new asymptotic value is an amplitude of opposite sign. When the pump phase is restored to its initial value a slow recovery of the acoustic amplitude is then observed.

The acoustic wave amplitude $Q$ cannot be measured by itself, so the next step is to evaluate its impact on the signal wave, which is the real measured quantity. This can be simply carried out by considering that the changes on the acoustic wave have only a small impact on the signal wave and can be treated as a first order linear perturbation. The change $\Delta Q$ on the acoustic amplitude induced by the brief $\pi$ phase shift on the pump wave can be expressed to first order like:

$$
\Delta Q \simeq 2 A_{p} A_{s}^{*} \Delta t
$$

In this expression the amplitudes are complex and the $\pi$ phase shift of the pump corresponds to a negative $A_{p}$, so that $\Delta Q$ is also negative. It is also interesting to point out that the acoustic loss $\Gamma$ is absent from this expression. Neglecting the propagation effects - which actually have an impact only during the transition and on the actual duration of the $\pi$ phase shift as seen by the signal wave - the corresponding change $\Delta A_{s}$ on the signal wave reads as a first approximation:

$$
\Delta A_{s} \simeq \frac{1}{2} g_{B} A_{p} \Delta Q^{*} \simeq g_{B}\left|A_{p}\right|^{2} A_{s} \Delta t
$$

The relative variations $\Delta A_{s} / A_{s}$ observed on the signal will thus only depend on the power of the pump and the duration $\Delta t$ of the $\pi$ phase shift. The acoustic lifetime has no impact on these variations! It must be pointed out that these variations $\Delta A_{s}$ are negative in a Brillouin gain configuration, so visualized like a "loss", and positive in a Brillouin loss configuration, so observed like a "gain". It is also important to pre-excite the acoustic wave to a large amplitude and using a low power $\mathrm{CW}$ wave or pre-pulse turns out to decrease the importance of the Brillouin echo.

The recovery of the acoustic wave after the $\pi$ phase shift will also cause a change of the signal amplitude. As a result of the reverse sign of the pump amplitude $A_{p}$ this change shows a behavior similar to the effect of the phase step, but on a time scale in the range of the acoustic lifetime $(\sim 10 \mathrm{~ns})$. This recovery effect is detrimental for the measurement and causes a delayed and relatively long-lasting overshoot on the signal amplitude. It will be discussed in the next section and a solution will be proposed to suppress it experimentally.

\section{EXPERIMENTAL DEMONSTRATION}

The Brillouin echoes were first observed using a simple modification of a classical pump and probe setup. The optical waves were both generated from the same laser, whose light was separated in two arms using a directional coupler. In one arm the probe wave was generated using the sideband technique ${ }^{3}$. In the other arm the pump was modulated by a phase modulator instead of the usual intensity modulator. The pump was thus circulating continuously at a constant power through the fiber under test. Short $\pi$ phase shifts were applied on the pump wave by driving the broadband phase modulator with short electrical pulses. We could observe immediately and very clearly the usual Brillouin signature on the time traces when the phase pulse propagated throughout the fiber and when the frequency difference between pump and probe was tuned on the Brillouin frequency. The spectral width of the Brillouin gain was equivalent to its natural linewidth and the effect of Brillouin echoes could be observed with phase pulse width down to 300 ps, which actually was the shortest pulse handled by our electrical pulse generator. Certainly shorter pulses would still be observed.

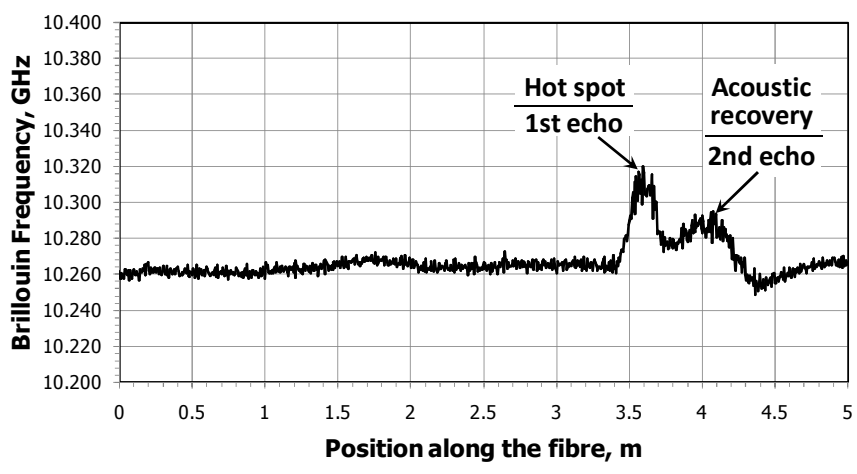

Fig. 2. Distribution of Brillouin frequency along an optical fibre obtained by applying a $10 \mathrm{~ns}$ long phase shift on the CW pump. A $20 \mathrm{~cm}$ hot spot is applied on the fiber, which is fully resolved. The effect of the acoustic wave recovery is also clearly observable as a broad delayed overshoot.

The total fiber length is $10 \mathrm{~m}$, the hot spot is $40 \mathrm{~K}$ above ambient temperature and the time traces were recorded with only 64 times averaging. CW pump power is $>500 \mathrm{~mW}$ and $\mathrm{CW}$ signal power $>100 \mathrm{~mW}$. 
Fig.2 shows a distributed measurement of the Brillouin frequency using a $1 \mathrm{~ns} \pi$ phase pulse along a fiber where a $20 \mathrm{~cm}$ hot spot $40 \mathrm{~K}$ above the ambient temperature is present. The hot spot is clearly resolved in terms of spatial and temperature resolutions. But the detrimental effect of the recovery of the acoustic wave is also clearly observed: it manifests through a spatially broader secondary echo, like a smoothed delayed replica of the hot spot.

We found a simple way to avoid this secondary echo, by hindering at the end of the phase pulse any coupling from the acoustic wave to the signal wave: this can be achieved by abruptly turning off the pump power at the end of the $\pi$ phase pulse. This actually makes the right term in Eq. 2 vanish and any change on the acoustic wave is no longer replicated on the signal wave. A time trace is shown in Fig. 3a using a $1.2 \mathrm{~ns} \pi$ phase pulse, showing sharp transitions and no smoothing effect due to the secondary echo. In Fig. 3b the 3-D graph of the Brillouin gain distribution clearly shows the well distinct $20 \mathrm{~cm}$ hot spot. These measurements were carried out in a two stage procedure: the pump power was turned on for $100 \mathrm{~ns}$ and the $\pi$ phase was applied at the very end of this pulse, during the last nanosecond. The long initial part of the pulse gives a classical Brillouin response with a broad spatial resolution of $10 \mathrm{~m}$. To extract the only contribution due to the Brillouin echo we then subtracted from the time trace the same measurement performed with no phase shift.
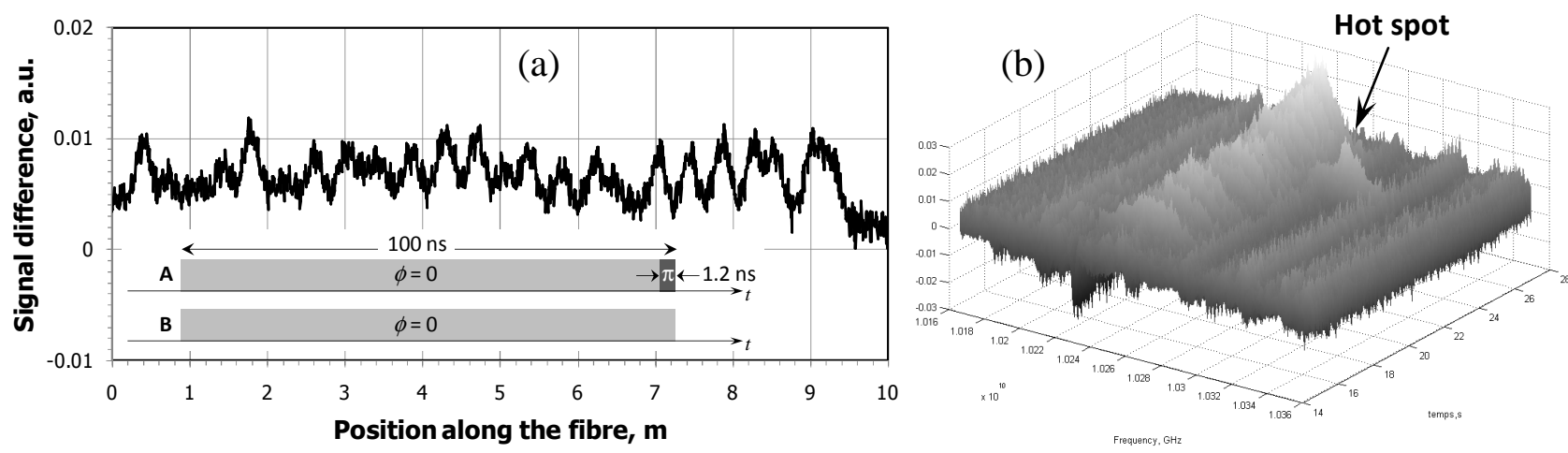

Fig. 3. (a) Difference between 2 sequential time traces, recorded successively: in configuration A (see insert) the long pulse activates the acoustic wave while the short ending $\pi$ phase pulse recalls the echo. The successive measurement in configuration B is used to subtract the contribution of the long pulse on the time traces. (b) Corresponding 3-D plot of the distribution of the Brillouin gain as a function of distance and frequency. The hot spot is clearly visible and well resolved in position and frequency.

\section{CONCLUSION}

In this paper we presented a physical description of the interaction based on Brillouin echoes that makes possible very high spatial resolution distributed measurements while maintaining the narrowband response of the natural Brillouin gain spectrum. In particular we could describe the experimental conditions that must be fulfilled to actually observe and exploit these Brillouin echoes. For the first time distributed measurements with a $10 \mathrm{~cm}$ spatial resolution are reported at $1550 \mathrm{~nm}$ using a semiconductor laser source. The technique shows potentialities to achieve spatial resolution in the $\mathrm{cm}$ range using a time domain technique, over distances that would be difficult to match using other approaches.

Acknowledgments: The authors are very grateful to Omnisens SA, Morges, Switzerland, for technical and financial support and to EPFL STI Seed fund program for its fellowship support.

\section{REFERENCES}

1. T.Horiguchi, T.Kurashima, M.Tateda, Photonics Tech. Lett., 2, p. 352, 1990.

2. X. Bao, D. J. Webb, and D. A. Jackson, Opt. Lett. 19, 141- (1994)

3. M. Niklès, L. Thévenaz, P.A. Robert, Opt. Lett. Vol 21, p 758-760. 1996.

4. A.Fellay, L.Thévenaz, M.Facchini, M.Niklès, Ph.Robert, Proceedings of12th International Conference on Optical Fiber Sensors, Williamsburg VA, OSA Publications, Washington DC, pp. 324-327, 1997.

5. X. Bao, A. Brown, M. DeMerchant, and J. Smith, Opt. Lett. 24, 510-512 (1999).

6. L. Zou, X. Bao, S. Afshar V., and L. Chen, Opt. Lett. 29, 1485-1487 (2004)

7. L. Zou, X. Bao, Y. Wan, and L. Chen, Opt. Lett. 30, 370-372 (2005)

8. V. P. Kalosha, E. Ponomarev, L. Chen, and X. Bao, Opt. Express 14, 2071-2078 (2006)

9. T. Horiguchi, R. Muroi, A. Iwasaka, K. Wakao and Y. Miyamoto, Proceeding of the $33^{\text {rd }}$ European Conference on Optical Communications (ECOC'2007), VDE Verlag GmbH, Berlin, Volume 5, Paper P018, pp.63-64 (2007). 